Nuclear Physics B146 (1978) 445-456

() North-Holland Publishing Company

\title{
JET PRODUCTION BY A GLUON SOURCE IN QCD *
}

\author{
Martin B. EINHORN \\ Randall Laboratory of Physics, University of Michigan, Ann Arbor, Michigan 48109, USA \\ and
}

Stanford Linear Accelerator Center, Stanford University, Stanford, California 94305, USA

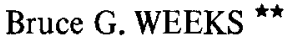

Randall Laboratory of Physics, University of Michigan, Ann Arbor, Michigan 48109, USA

Received 16 August 1978

The properties of jets produced by a gluon source are examined in QCD. We give a simple derivation of the jet opening angle (up to an undetermined constant) using arguments of general applicability. At asymptotic energies, gluon jets are found to be wider than quark jets in an intuitively natural way. Unfortunately, in the energy range anticipated for PEP or PETRA, the results are quite sensitive to variations in the undetermined constant so that firm quantitative predictions cannot be made within the present approximations.

\section{Introduction}

Reasonably direct experimental confirmation of the existence of quarks is found in the decay schemes of heavy quarkonium states, in deep inelastic scattering, and in the observation of jets in $\mathrm{e}^{+} \mathrm{e}^{-}$annihilation $\star \star \star$. However, while these results are suggestive of an asymptotically free field theory, the precise details of such a theory remain unconfronted by experiment. In particular, existing data shed little light on the nature of those partons which are not quarks.

That perturbation calculations can be applied to strong interactions is the result of fairly recent theoretical developments. Because of asymptotic freedom, the running coupling constant decreases with increasing energy. Nevertheless, many cross sections cannot be calculated perturbatively at high energies due to mass singularities as $m / E \rightarrow 0$. Recently, Sterman and Weinberg [2] have stressed the impor-

* Supported by the Department of Energy.

$\star$ Partially supported by a University of Michigan Rackham Predoctoral Fellowship.

$\star \star \star$ For recent reviews of this evidence, see ref. [1]. 
tance of cross sections which are free of such singularities and for which one may take the quark masses to be zero at the outset. They have suggested that such cross sections might be susceptible to direct experimental confirmation, despite complications associated with confinement of quark and gluons. This has stimulated a variety of applications $\star$.

In ref. [2], the production of $N$-jet final states in $\mathrm{e}^{+} \mathrm{e}^{-}$annihilation was discussed. Here, and henceforth in this paper, the number of jets in a final state of total energy $E$ is counted by neglecting soft particles (with energy $<\epsilon E$ ) and grouping hard particles with angular separation less than $2 \delta$ in the same jet ${ }^{\star}$. By considering the dependence of the 2-jet production cross section on $\delta$ and $\epsilon$ in a manner reviewed below, Sterman and Weinberg suggest that one can understand in detail the width of jets and, conversely, use data on jets to test QCD. They define an angle for quark jets $\delta_{\mathrm{q}}(E)$ (see eq. (5) below) produced in $\mathrm{e}^{-} \mathrm{e}^{+}$annihilation, which they compute perturbatively to order $g^{2}$ for $\delta, \epsilon<<1$. To that order, $\delta_{\mathrm{q}}(E)$ differs from zero because of gluon bremsstrahlung from the quark-antiquark pair.

In this paper, we apply QCD to the analysis of gluon jet production. Detailed experimental confirmation of our results would be strong evidence for the vector gluon nature of QCD. Unfortunately, it turns out that at energies available at accelerators such as PEP or PETRA, one will have to go beyond the approximations used here to perform quantitative tests.

Normally, the primary source of gluon production in $\mathrm{e}^{-} \mathrm{e}^{+}$annihilation is hard radiation from light quarks, as discussed in detail in ref. [4]. To understand how the gluon jets are smeared out in a manner analogous to the calculation of ref. [2], one would calculate the soft or approximately collinear corrections to this three-jet process. This requires going to next order in perturbation theory, a formidable (though not impossible) task. Even then, one would have to discuss a way to differentiate gluon jets from quark jets. However, in the decays of heavy quark bound states, it is believed that gluons are produced directly from quark-antiquark annihilation. Thus, for example, the total decay rate into hadrons of $0^{-+}, 0^{++}, 2^{++}$, etc., bound states (such as $\eta_{\mathrm{c}}$ ) is thought to be via two gluons $[5,6]$. The hadronic decay of $1^{--}$states such as $\psi(3.1)$ or $T(9.4)$ is thought to be predominantly via three gluons [6-8]. Certain radiative inclusive decays such as $T^{\prime} \rightarrow \gamma+$ hadrons may be expected to yield two gluon jets $[8,9]$ ". Higgs bosons may also lead to two gluon jets [10]. This suggests that the investigation of jet production from a local source of gluons might be more than an academic exercise. Since gluons carry a higher color charge than quarks, we can anticipate that they will bremsstrahlung more efficiently and be more dispersed. Consequently, a gluon jet may be broader than a quark jet at the

* For examples, see ref. [3].

$\star \star$ The cross section for two jets, so defined, differs slightly from the definition used in ref. [2] which specified a fixed detector. For a $4 \pi$ detector, our definition seems more natural. For $\delta$ and $\epsilon$ sufficiently small, the two definitions agree for $\delta>\epsilon$.

$\star \star \star$ The investigation of the even-charge conjugation channel by radiative decays of narrow, odd- $C$ resonances in $\mathrm{e}^{-\mathrm{e}^{+}}$annihilation was suggested in ref. [9a $]$. 
same energy, and this expectation is borne out by our calculation. Experimentally, this might be studied by comparing the jets observed (if any) from a series of resonances (bottomonium, toponium, ...).

In a slightly more speculative vein, we remark that it may also be possible to study gluon jets in hadron collisions although meaningful investigations presumably await the construction of new $p p$ or $p \bar{p}$ colliding beam facilities.

The outline of the paper is as follows. In sect. 2, we review the situation for quark jets, stressing the limited quantitative validity of theoretical results so far. In sect. 3, we derive a result for gluon jets analogous to ref. [2] in order to identify how bremsstrahlung by gluons will differ from bremsstrahlung by quarks. Sect. 4 contains a discussion of this results. Finally, in sect. 5 , we conclude with some comments on confinement and the parton model and on the implications of our conclusions for future work. In the appendix, we present some mathematical details of the derivation of the result in sect. 3 .

\section{Review of quark jets}

The Sterman-Weinberg result for the two-jet production cross section to order $g^{2}$ is [2]

$$
\sigma_{0}\left\{1-\frac{4 \alpha_{s}}{\pi} C_{2}(R)\left[\ln \frac{1}{\delta}\left(\ln \frac{1}{2 \epsilon}-\frac{3}{4}\right)+\frac{\pi^{2}}{12}-\frac{5}{8}\right]\right\},
$$

where

$$
\sigma_{0}=\frac{4}{3} \frac{\pi \alpha^{2}}{E^{2}} N_{\mathrm{c}} \sum Q_{i}^{2}
$$

is the zeroth-order total cross section; $N_{\mathrm{c}}$, the number of quark colors; $Q_{i}$, the charge of quarks of flavor $i$. In eq. (1), $C_{2}(R)$ is the value of the quadratic Casimir operator for the fermion representation ${ }^{\star}\left(C_{2}(R)=\frac{4}{3}\right.$ for the $\mathrm{SU}(3)$ fundamental representation). Eq. (1) represents the first terms of an expansion in $\delta$ and $\epsilon$ in which terms vanishing with $\delta, \epsilon \rightarrow 0$ have been dropped.

Since the total cross section to order $g^{2}$ is [12]

$$
\sigma_{0}\left(1+\frac{3}{4} C_{2}(R) \frac{\alpha_{\mathrm{s}}}{\pi}\right)
$$

the fraction $f$ of events consisting of two jets may be written as

$$
1-f=\frac{4 \alpha_{s}}{\pi} C_{2}(R)\left[\ln \frac{1}{\delta}\left(\ln \frac{1}{2 \epsilon}-\frac{3}{4}\right)+\frac{\pi^{2}}{12}-\frac{7}{16}\right] .
$$

\footnotetext{
* Our notation for Casimir operators follows ref. [11].
} 
Table 1

(a) Fraction $f$ (in percent) of events for various values of $\epsilon$ and $\delta$ (in degrees), (b) Value of $\ln \delta^{-1}\left(\ln (1 / 2 \epsilon)-\frac{3}{4}\right)$

\begin{tabular}{lllllll}
\hline$\epsilon / \delta$ & 1 & 5 & 10 & 15 & 20 & 30 \\
\hline (a) & & & & & & \\
0.05 & $<0$ & $<0$ & 3 & 29 & 42 & 59 \\
0.1 & $<0$ & 28 & 45 & 56 & 63 & 72 \\
0.15 & 35 & 57 & 66 & 71 & 75 & 81 \\
0.2 & 69 & 77 & 81 & 82 & 83 & 86 \\
(b) & & & & & & \\
0.05 & 6.28 & 3.79 & 2.71 & 2.08 & 1.63 & 0.56 \\
0.1 & 3.48 & 2.10 & 1.50 & 1.15 & 0.90 & 0.56 \\
0.15 & 1.84 & 1.11 & 0.79 & 0.61 & 0.48 & 0.29 \\
0.2 & 0.67 & 0.41 & 0.29 & 0.22 & 0.18 & 0.11 \\
\hline
\end{tabular}

It is apparent from the preceding discussion that the constant term in (3) is dependent upon the precise nature of the source (in this case, a vector electromegnetic current). On the other hand, the terms divergent as $\delta$ or $\epsilon$ tend to zero are presumably source independent (although we have not proved this rigorously ${ }^{\star}$ ) and are characteristic of QCD itself. Recall that eq. (1) and, hence, eq. (3) are obtained by neglecting terms of $\mathrm{O}(\delta)$ or $\mathrm{O}(\epsilon)$ and higher. Their regime of applicability, therefore, requires $\delta$ and $\epsilon$ sufficiently small, presumably so that the logarithmic terms are larger than the constant terms. Yet, to apply perturbation theory, $\alpha_{\mathrm{s}}$ must be sufficiently small so that the corrections to the cross section (eq. (1)) or to $f$ (eq. (3)) are small. Even at the highest energies anticipated in the not-too-distant future for PEP or PETRA, these approximations are marginal.

In table 1 a, we evaluate eq. (3) for various values of $\epsilon$ and $\delta$ for a c.m. energy $E=$ $30 \mathrm{GeV}$. In so doing, we have ulilized the asymptotic QCD formula [6]

$$
\frac{\alpha_{\mathrm{s}}}{\pi}(E)=\frac{6}{\left(33-2 N_{\mathrm{f}}\right) \ln (E / \Lambda)},
$$

with a scale $\Lambda$ for the logarithm taken to be $0.5 \mathrm{GeV}$. However, the number of quark flavors $N_{\mathrm{f}}$ to be included here is ambiguous at finite energies. Presumably, we should include those flavors for which the quark mass is negligible at any given energy. We will assume that only the lightest three flavors are to be included for the range of energies of interest ${ }^{\star \star}$.

* We have in mind that the soft or collinear corrections to a non-infrared divergent process may be calculable from an analog of the low-energy theorem of QED given by Low [15]. This analogy suggests that perhaps the constant term in eq. (1) is also source independent。

$\star \star$ To some extent this can be resolved experimentally by observing how frequently the jets detected contain the heavier quarks. 
For $E=30 \mathrm{GeV}, \alpha_{\mathrm{s}} / \pi$ is quite small (0.06), so one would expect perturbation theory to be good. However, perusal of table 1 a shows that we are caught in a bind. To justify the expansion in $\epsilon$ and $\delta$, we must choose them small. Yet the smaller we choose them, the larger the correction term, i.e., the more questionable is the perturbation expansion. Clearly, the formula makes sense only for $\ln (1 / 2 \epsilon)>\frac{3}{4}(\epsilon<$ 0.236 ) and for $\delta$ sufficiently small so that the log term is larger than the constant term. In table $1 \mathrm{~b}$, we list the value of

$$
\ln \frac{1}{\delta}\left(\ln \frac{1}{2 \epsilon}-0.75\right)
$$

which is to be compared with the constant term $\frac{1}{12} \pi^{2}-\frac{7}{16} \approx 0.38$. Apparently, the range of validity of this formula is barely marginal at this energy. Because $\alpha_{\mathrm{s}}$ decreases only logarithmically with energy, the applicability of eq. (3) improves only very slowly with increasing energy.

A more sensitive test of the energy dependence was suggested in ref. [1]. At any given energy $E$, what opening angle $\delta_{\mathrm{q}}(E)$ is required so that some predetermined fraction $f$ of the events have at least $1-\epsilon$ of their energy within the solid angle defined by $\delta_{\mathrm{q}}(E)$ ? Solving eq. (3) for $\delta$ yields at asymptotic energies the power law

$$
\delta_{\mathrm{q}}(E)=E^{-\frac{(1-f)}{8}\left(\frac{33-2 N_{\mathrm{f}}}{4 \ln (1 / 2 \epsilon)-3}\right)} .
$$

For $N_{\mathrm{f}}=3$ and $\epsilon=0.1$, this gives $E^{0.98(1-f)}$.

One cannot expect this asymptotic formula to work at finite energies any better than eq. (3) itself. Inclusion of the constant term is given by the substitution in the exponent of

$$
1-f \rightarrow 1-f-\frac{\alpha_{\mathrm{s}}}{\pi} C_{2}(R)\left(\frac{1}{3} \pi^{2}-\frac{7}{4}\right) .
$$

The correction term is of magnitude 0.11 for $E=30 \mathrm{GeV}$ and represents a very significant change. It would be fortuitous if the uncalculated corrections to eq. (3) were negligible. Since the failure of eq. (3) is primarily due to the expansion in $\delta$ and $\epsilon$, it should not be difficult to obtain a more reliable formula by calculating the next few terms in the expansion ${ }^{\star}$.

\section{Gluon jet derivation}

In this section, we will derive a formula for gluon jets analogous to eq. (3). Before getting into details, we can anticipate some features of the result. Bremsstrahlung by a gluon will be proportional to the "color charge" $C_{2}(G)$ of a gluon, just as the corrections in eq. (3) were proportional to the quark "charge" $C_{2}(R)$. Since for $\mathrm{SU}(3)$ color, $C_{2}(G)=3$ while $C_{2}(R)=\frac{4}{3}$, we are likely to find a much larger effect.

* Elsewhere, onc of us (BGW) presents the exact calculation of the fraction $f$ to first order in $\alpha_{\mathrm{s}}$ See ref. [17]. 
In addition, a gluon may split into quark-antiquark pairs. Altogether then, resolving a gluon jet may well require much higher energies then resolving a quark jet. As a first step toward quantifying these qualitative remarks, we derive a formula for the opening angle of a gluon jet $\delta_{\mathrm{q}}(E)$ analogous to eq. (5) for a quark jet. Therefore, we compute in this section to order $g^{2}$ the two-jet production rate from a gluon source ${ }^{\star}$ which we choose to be $\left(F_{\mu \nu}^{a}\right)^{2}$.

The remainder of this section is devoted to sketching the derivation of the result exhibited in eq. (9), to which the reader uninterested in details may pass. We define the following production rates:

$W_{0} \equiv$ the total rate to order $g^{0}$;

$W^{2} \equiv$ the order $g^{2}$ contribution to the 2 -jet production rate;

$W^{3} \equiv$ the order $g^{2}$ contribution to the 3 -jet production rate.

The number of jets in the final state is defined exactly as in sect. 1 and should not be confused with the number of quarks or gluons in the final state. To this order, the total production rate is simply the sum $W_{\text {tot }}=W_{0}+W^{2}+W^{3} . W^{2}$ receives contributions from infrared divergent pieces, viz., the virtual corrections to the twogluon final state plus the soft or nearly collinear configurations in the three-gluon state. However, since $W_{\text {tot }}, W_{0}$, and $W^{3}$ are all infrared finite, it follows that $W^{2}$ is also not divergent, as expected. Note that the fraction of two-jet events may be written to this order as

$$
f \equiv \frac{W_{0}+W^{2}}{W_{0}+W^{2}+W^{3}} \approx 1-\frac{W^{3}}{W_{0}} .
$$

Thus, $f$ may be calculated directly from $W^{3}$ and $W_{0}$ without encountering any divergences. In particular, there is no need for infrared regularization ${ }^{\star \star}$.

We proceed to calculate $W^{3}=\int \mathrm{d} f(2 \pi)^{4} \delta\left(p_{f}-p_{i}\right)|T|^{2}$ to order $g^{2}$, where the phase-space integration includes all 3-jet events. In the limit $\delta, \epsilon \rightarrow 0, \mathrm{~d} f$ includes all of 3-particle phase space, and $W^{3}$ is divergent. This is due to bremsstrahlung, as follows: if particle 1 is emitted by particle 2 , the associated Feynman graph has the propagator $1 /\left(p_{1}+p_{2}\right)^{2}=1 / 2 p_{1} p_{2}(1-\cos \Delta)$, where $\Delta$ is the angle between $p_{1}$ and $p_{2}$ and $p_{i}$ is used to denote both a 4 -vector and its zero component. The denominator displays both infrarea $\left(p_{i} \rightarrow 0\right)$ and collinear $(\Delta \rightarrow 0)$ singularities, and the $\mathrm{d} f$ integral would diverge. However, for $\delta, \epsilon>0$, all singular points are excluded from the $\mathrm{d} f$ integration, and $W^{3}$ is finite.

Performing the $\mathrm{d} f$ integral is technically difficult for two reasons. First, the integrand is a priori a complicated function of the phase space variables, although it

* With slight generalization, the following derivation applies to and yields the same result (eq. (9)) for any Lorentz- and gauge-invariant local source of gluon pairs with non-vanishing $g^{\mu \nu}$ term so long as the source produces more than two gluons only at the expense of additional powers of $g$.

* Precisely analogous remarks apply to the derivation of eq. (3) for quark jets and can be used instead of the method given in ref. [2]. In essence, eqs. (1) and (3) can be obtained by subtracting the cross section for three jets (given in ref. [4]) from the total cross section. 


$$
\begin{aligned}
& \text { num } \frac{i g^{\mu_{1} \mu_{2}}}{k^{2}} \\
& \bullet \longleftrightarrow \frac{i}{\gamma \cdot p} \\
& \sum_{j}^{i} \operatorname{lig}_{\mu}^{\mu} \frac{\lambda_{i j}^{a}}{2} \\
& g f_{a_{1} a_{2} a_{3}}\left[g \mu^{\mu_{1} \mu_{2}}\left(p_{2}-p_{1}\right)^{\mu_{3}+g} \mu_{2} \mu_{3}\left(p_{3}-p_{2}\right)^{\mu_{1}+g} \mu_{3} \mu_{1}\left(p_{1}-p_{3}\right)^{\mu_{2}}\right] \\
& 2^{v^{2}}+x^{3}+3
\end{aligned}
$$

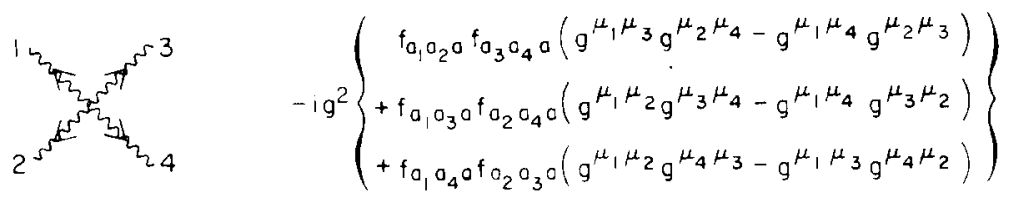

$$
\begin{aligned}
& \ln _{x x^{n}} n^{n^{2}} \quad i\left[g^{\mu_{1} \mu_{2}} p_{1} \cdot p_{2}-p_{1}^{\mu_{2}} p_{2}^{\mu_{1}}\right] \\
& \text { 12 } \sum^{2} a^{2}
\end{aligned}
$$

Fig. 1. Relevant Feynman rules for QCD with source $\left(F_{\mu \nu}^{a}\right)^{2}$.

might be that, as in the Sterman-Weinberg calculation, the integrand simplifies drastically under an appropriate choice of variables. Second, the limits of the $\mathrm{d} f$ integration corresponding to 3 -jet final states are not simple. However, in this paper we compute $W^{3}$ only up to an undetermined contribution which is finite as $\delta, \epsilon \rightarrow 0$. Thus, we are free to make approximations whose errors terms remain finite in that limit. As a result, the calculation turns out to be very easy.

Our Feynman rules are given in fig. 1. For now, we neglect quarks. Then, the only singular contributions to $T$ are graphs of the form shown in fig. 2. Although the singularity structure of the propagator looks like $1 / p_{1} p_{2} \Delta^{2}, T$ itself has a collin-

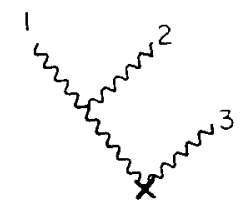

Fig. 2. Singular contribution to $T$. 
ear singularity going like $1 / \Delta$. This is because every term in the numerator of $T$ has a factor of $\epsilon_{i} \cdot p_{j}$, which is of order $\Delta$ for $\Delta$ small. (Here, we take advantage of the fact that we may choose the gluon polarization to satisfy $\epsilon_{i}^{0}=0, \epsilon_{i}^{*}=\epsilon_{i}$, and $\varepsilon_{i} \cdot p_{i}=$ 0.)

Thus, the singularity structure of $T$ looks like $\left(p_{1} p_{2} \Delta\right)^{-1}$. But, as shown in the appendix, phase space contains the factor $p_{1} p_{2} \sin \Delta$. It follows that a cross term contribution to $W^{3}$ consisting of the product of a singular and a non-singular graph is non-divergent as $\delta, \epsilon \rightarrow 0$ and can be neglected. Therefore, we need only consider products of singular graphs.

The details of the remainder of the calculation of $W^{3}$ may be found in the appendix. The result is

$$
1-f=\frac{4 \alpha_{s}}{\pi} C_{2}(G)\left[\ln \frac{1}{\delta}\left(\ln \frac{1}{2 \epsilon}-\frac{11}{12}\right)+r(\delta, \epsilon)\right],
$$

where the remainder $r(\delta, \epsilon)$ is finite in the limit $\delta, \epsilon \rightarrow 0$.

As expected, the formula is quite similar to eq. (3) except that $C_{2}(R)$ for quarks has been replaced by $C_{2}(G)$ for gluons. Since $C_{2}(G) / C_{2}(R)=\frac{9}{4}$, we can immediately conclude that the expansion in $\delta$ and $\epsilon$ will be valid only at extremely high energies.

So far we have neglected quarks. Their inclusion in the calculation of $W^{3}$ involves no new ideas, and we obtain

$$
1-f=\frac{4 \alpha_{\mathrm{s}}}{\pi}\left[C_{2}(G) \ln \frac{1}{\delta} \ln \frac{1}{2 \epsilon}-\left(\frac{11}{12} C_{2}(G)-\frac{1}{8} N_{\mathrm{f}} C_{2}(R) \ln \frac{1}{\delta}\right],\right.
$$

neglecting terms which are finite as $\delta, \epsilon \rightarrow 0$. This is the desired analog of (3). Notice that the constant coefficient of $\ln (1 / \delta)$ is precisely the factor occurring in the $\beta$ function which determines $\alpha_{\mathrm{s}}(E)[6]$.

\section{Discussion of results}

Given our caveats concerning the applicability of the formula for quarks (eq. (3)), the reader will have no difficulty appreciating that this situation is even worse for gluon jets. From eq. (8), we see that we must now choose $\epsilon<0.2$ to obtain a meaningful expansion in $\epsilon$. Unfortunately, now we have not calculated the constant additional term so it is impossible to estimate how small $\delta$ must be chosen. As before, we expect that for a range of values of $\delta$ and $\epsilon$ sufficiently small to justify neglecting the constant term, $f$ will not be near 1 , signifying a breakdown of perturbation theory. (Indeed, for $\epsilon=0.1$ and $\delta \leqslant 30^{\circ}$, we find $f \leqslant 64 \%$ for $E=30 \mathrm{GeV}$.) Consequently, we suspect that eq. (9) is quantitatively useless until extraordinarily high energies.

As before, it is interesting to "solve" for $\delta$ to obtain the asymptotic power law

$$
\delta_{\mathrm{q}}(E)=E^{-\frac{(1-f)}{18}\left(\frac{33-2 N_{\mathrm{f}}}{4 \ln (1 / 2 \epsilon)-\left(33-2 N_{\mathrm{f}}\right) / 9}\right)} .
$$


This suggests that the collimation of gluon jets will shrink much more slowly than for quark jets. For $N_{\mathrm{f}}=3$ and $\epsilon=0.1$, we find $E^{-0.44(1-f)}$.

For $N_{\mathrm{f}}=3$, the correction to the $\ln 2 \epsilon$ term is fortuitously exactly the same as in eq. (5), so we may say that ${ }^{\star}$

$$
\delta_{\mathrm{g}}(E)=\delta_{\mathrm{q}}(E)^{4 / 9},
$$

where, it must be noted again, the exponent is simply the ratio $C_{2}(R) / C_{2}(G)$ of "color charges" for the quarks and gluons, respectively.

\section{Concluding remarks}

In this paper, no mention has been made of the non-perturbative confinement mechanism which prevents the quarks and gluons themselves from emerging as asymptotic states. The interpretation of the perturbative calculations as predictions for physical cross sections rests on certain, often unstated assumptions about the nature of the confining mechanism. We follow the usual parton-model hypotheses by supposing that each quark or gluon of large momentum leads to a jet of hadrons characterized by a sharply damped, transverse-momentum distribution; typically, the mean transverse momentum $\left\langle p_{\perp}\right\rangle$ is $500-800 \mathrm{MeV}$. It is further assumed that this non-perturbative transverse-momentum cutoff is essentially independent of the large momentum of the original quark or gluon. It is supposed to approach a finite value in the infinite momentum limit of the parent parton ${ }^{\star \star}$.

This means that a parton of momentum $P$ will yield a jet of hadrons with a nonperturbative opening angle $\Delta \sim\left\langle p_{\perp}\right\rangle / P$, which must be distinguished from the angle $\delta_{\mathrm{q}}$ or $\delta_{\mathrm{g}}$ given in eqs. (5) or (10). In our discussion we have tacitly assumed that $\delta>\Delta$. At finite energies, this gives a lower limit on the range of applicability of the perturbative formula. For example, for a parton of momentum $15 \mathrm{GeV}$, we find $\Delta \approx 3^{\circ}$.

From the parton point of view, the angular dependence on $\delta$ discussed in this paper or in ref. [2] should not be thought of as the spreading of a jet but rather the extent to which, at any given energy, three embryonic jets will be confused as two.

We would like to conclude by reemphasizing that, at energies of experimental interest, the expansion in $\epsilon$ and $\delta$ given in eqs. (3) and (9) are invalid. Since the higher-order correction to the total cross section is quite small (see eq. (2)), a perturbation theory calculation of $1-f$ should be valid for large enough $\epsilon$ and $\delta$. Consequently, it seems worthwhile, as a test of QCD and the confinement hypotheses here, to recalculate $1-f$ in $\mathrm{e}^{-} \mathrm{e}^{+}$annihilation without expanding in $\epsilon$ and $\delta$. This calculation should not be difficult and will be presented in a subsequent publication [17].

${ }^{\star}$ Of course, by the energy range where eq。 $(11)$ is valid, we anticipate $N_{\mathrm{f}}$ considerably larger than 3.

$\star \star$ We have tacitly assumed that quarks and gluons fragment into hadrons in essentially the same way. Alternative possibilities have been suggested, e.g., in ref. [16]. 
We would like to thank R.N. Cahn, J. Ellis, C.-K. Lee, and Y.P. Yao for helpful conversations. During the course of this work, we became aware that this problem was also being considered by K. Shizuya and S.-H.H. Tye [13], and we would like to thank both these authors for discussions and for communicating their results to us prior to publication. We would like to thank C. Quigg for extending to us the hospitality of the Theory Group at Fermilab, where portions of this work were per. formed.

\section{Appendix}

Here we present the remaining details of the calculation of $w^{3}$. Using covariant normalization,

$$
\mathrm{d} f \equiv \frac{1}{3 !} \prod_{i=1}^{3} \frac{\mathrm{d} p_{i}}{(2 \pi)^{3} 2 p_{i}}
$$

Some typical kinematical manipulations yield

$$
\int \mathrm{d} f(2 \pi)^{4} \delta\left(p_{f}-p_{i}\right)=\frac{1}{24} \frac{1}{(2 \pi)^{4}} \frac{p_{1} p_{2}}{\left|\partial p_{3}^{2} / \partial p_{2}\right|_{p_{3}^{2}=0}} \mathrm{~d} \Omega_{p_{2}} \mathrm{~d} p_{1} \mathrm{~d} \Delta \sin \Delta,
$$

where $\Delta$ is the angle between $p_{1}$ and $p_{2}$. Due to the particle interchange symmetry of $|T|^{2}$, we can restrict $p_{1}<p_{2}<p_{3}$ in the $\mathrm{d} f$ integral and multiply by 6 without changing $W^{3}$. The resulting contribution to phase space in the limit $\delta, \epsilon \rightarrow 0$ is enclosed by the dashed line in fig. 3 . However, we can safely approximate this

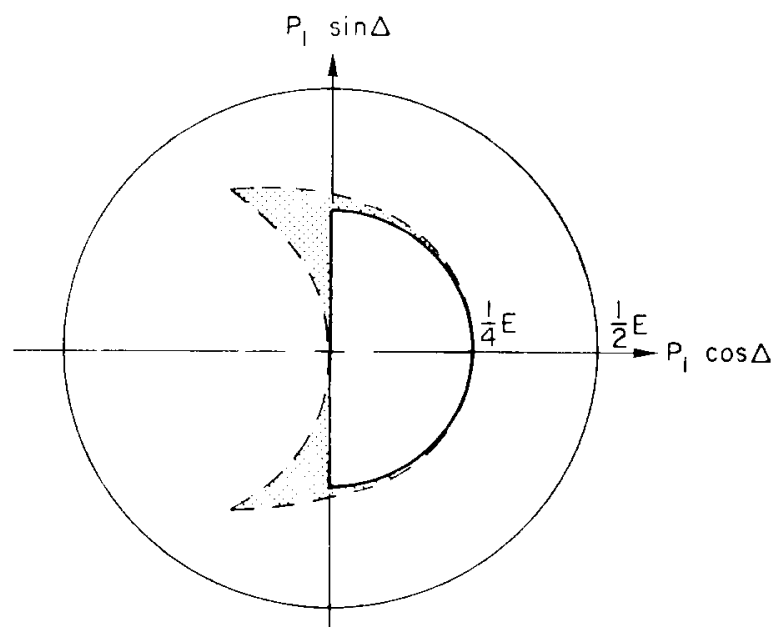

Fig. 3. Phase-space subregions of interest. 
region by the semicircular region enclosed by the solid line in the same figure. This is because the resulting error in the limit $\delta, \epsilon \rightarrow 0$ (already taken in the figure) is just the integral over the shaded region. But, as observed earlier, the full integrand goes at worst like $1 / p_{1} \Delta$ and clearly yields a finite integral over the shaded region.

Therefore, excluding the two-jet phase space from the semicircular region, we may take our limits of integration to be $\epsilon E<k<\frac{1}{4} E$ and $2 \delta<\Delta<\frac{1}{2} \pi$.

Since we only need consider singular graphs such as in fig. 2 ,

$$
T=\sum g f_{a_{1} a_{2} a_{3}} \frac{E p_{3}}{p_{1} \cdot p_{2}}\left[-\epsilon_{1} \cdot \epsilon_{3} p_{1} \cdot \epsilon_{2}+\epsilon_{2} \cdot \epsilon_{3} p_{2} \cdot \epsilon_{1}+\epsilon_{1} \epsilon_{2} p_{1} \cdot \epsilon_{3}\right],
$$

where the sum runs over the 3 cyclic permutations of the indices and where we have used relations such as $p_{2} \cdot \epsilon_{3}=-p_{1} \cdot \epsilon_{3}$.

Furthermore, we need only keep those terms which have singularities in the semicircular region, i.e.,

$$
\begin{aligned}
& g f_{a_{1} a_{2} a_{3}}\left\{\frac{E p_{3}}{p_{1} \cdot p_{2}}\left[-\epsilon_{1} \cdot \epsilon_{3} p_{1} \cdot \epsilon_{2}+\epsilon_{1} \cdot \epsilon_{2} p_{1} \cdot \epsilon_{3}\right]\right. \\
& \left.\quad+\frac{E p_{2}}{p_{3} \cdot p_{1}} \epsilon_{3} \cdot \epsilon_{2} p_{2} \cdot \epsilon_{1}+\frac{E p_{3}}{p_{1} \cdot p_{2}} \epsilon_{3} \cdot \epsilon_{2} p_{2} \cdot \epsilon_{1}\right\} \equiv A+B+C
\end{aligned}
$$

In computing $|T|^{2}$, we note that $A^{2}+2 A C$ has collinear divergences as $\delta, \epsilon \rightarrow 0$, $B^{2}+2 B C$ has infrared divergences, $C^{2}$ has both, and $2 A B$ has neither and can be dropped.

Now, $p_{1} \cdot p_{2}, p_{1} \cdot p_{3}, p_{1} \cdot \epsilon_{3}, \epsilon_{1} \cdot \epsilon_{3}$, and $\left|\partial p_{3}^{2} / \partial p_{2}\right|_{p_{3}^{2}=0}$ are all fairly complicated functions of $p_{1}$ and $\Delta$. However, considering $p_{2}$, note that the difference $p_{2}-\frac{1}{2} E-p_{1}$ vanishes both with $p_{1}$ and $\Delta$. Thus error terms resulting from the replacement $p_{2} \rightarrow \frac{1}{2} E-p_{1}$ all have a power of $p_{1} \Delta$ appearing which eliminates any collinear or IR divergences present. Extending this reasoning for various cases, we find that we can make the replacements $p_{3}=\frac{1}{2} E, p_{1} \cdot p_{3}=p_{1} p_{3}(1+\cos \Delta), p_{1} \cdot \epsilon_{3}=$ $-2 p_{1} p_{2} \sin \Delta / E, \epsilon_{i} \cdot \epsilon_{j}=0$ or \pm 1 , and $\left|\partial p_{3}^{2} / \partial p_{2}\right|_{p_{3}^{2}=0}=2 E$, as well as $p_{2}=\frac{1}{2} E-p_{1}$.

It is now straightforward to sum $|T|^{2}$ over the final gluon polarizations:

$$
\sum_{\lambda_{1} \lambda_{2} \lambda_{3}}|T|^{2}=g^{2}\left(f_{a b c}\right)^{2} \frac{1}{2} E^{4}\left\{\frac{4}{p_{1}^{2}} \frac{1}{\sin ^{2} \Delta}+\left[\frac{1}{p_{2}^{2}}+\frac{1}{\left(\frac{1}{2} E\right)^{2}}\right]-\frac{\sin ^{2} \Delta}{(1-\cos \Delta)^{2}}\right\} \text {. }
$$

The integrals are now trivial, yielding eq. (8).

\section{Note added}

A preprint has appeared [14] which determines the corrections to eq. (3) to order $\delta^{2}, \epsilon^{2}$, by calculating $W^{3}$ in a manner analogous to our calculation in the gluon case. 


\section{References}

[1] J.D. Jackson, in Proc. SLAC Summer Inst. on particle physics, August 1976 (LBL-5500mc, August 1976);

G.J. Feldman, Banff Summer Inst. on particles and fields, 1977 (SLAC-PUB-2068, December 1977);

T. Appelquist, R.M. Barnett and K. Lane, Stanford Linear Accelerator Center preprint SLAC-PUB-2100 (March 1978);

G. Hanson, 13th Rencontre de Moriond, 1978 (SLAC-PUB-2118, May 1978).

[2] G. Sterman and S. Weinberg, Phys. Rev. Lett. 39 (1977) 1436.

[3] C.L. Basham, L.S. Brown, S.D. Ellis and S.T. Love, Phys. Rev. D17 (1978) 2298;

E. Farhi, Phys. Rev. Lett. 39 (1977) 1587;

H. Georgi and M. Machacek, Phys. Rev. Lett. 39 (1977) 1237;

A. De Rujula, J. Ellis, E.G. Floratos and M.K. Gaillard, Nucl. Phys. B138 (1978) 387;

S.-Y. Pi, R.L. Jaffe and F.E. Low, Phys. Rev. Lett. 41 (1978) 142.

[4] J. Ellis, M.K. Gaillard and G.G. Ross, Nucl. Phys. B111 (1976) 253.

[5] A. De Rujula, J. Ellis, E.G. Floratos and M.K. Gaillard, see ref. [3].

[6] T. Appelquist and H.D. Politzer, Phys. Rev. Lett. 34 (1975) 43; Phys. Rev. D12 (1975) 1404.

[7] T.A. DeGrand, Y.J. Ng and S.-H.H. Tye, Phys. Rev. D16 (1977) 3521.

[8] S.J. Brodsky, T.A. DeGrand, R.R. Horgan and D.G. Coyne, Phys. Lett. 73B (1977) 203;

H. Fritzsch and K.-H. Streng, Phys. Lett. 74B (1978) 90;

K. Koller and T. Walsh, DESY preprint 78/16 (March 1978).

[9] M. Krammer and H. Krasemann, Phys. Lett. 73B (1978) 58;

P. Roy and T.F. Walsh, DESY preprint 78/28 (June 1978);

M. Krammer, Phys. Lett. 74B (1978) 361.

[9a] M.B. Einhorn and M.J. Creutz, Phys. Rev. Lett. 24 (1970) 341.

[10] F. Wilczek, Phys. Rev. Lett. 39 (1977) 1304.

[11] D.J. Gross and F. Wilczek, Phys. Rev. D8 (1973) 3633.

[12] T. Appelquist and H. Georgi, Phys. Rev. D8 (1973) 4000;

A. Zee, Phys. Rev. D8 (1973) 4038.

[13] K. Shizuya and S.-H.H. Tye, Fermilab preprint Fermilab-Pub-78/54-THY (June 1978).

[14] P.M. Stevenson, Imperial College preprint ICTP/77-78/25 (July 1978).

[15] F.E. Low, Phys. Rev. 110 (1958) 974.

[16] G.L. Kane and Y.-P. Yao, University of Michigan preprint UM-HE-77-44 (1977).

[17] B.G. Weeks, University of Michigan preprint UM-HE-78-49 (1978). 